\title{
Special Education: Clinical Application of Chinese Version Sensory Profile
}

\author{
Hong Tang ${ }^{1}$, Man C.M. Tsui ${ }^{2}$, Brian H.C. Cheung ${ }^{2}$ \& Guofu Zhang ${ }^{1}$ \\ ${ }^{1}$ Wuxi Mental Health Center, Wuxi Tongren International Rehabilitation Hospital, Nanjing Medical University, \\ China \\ ${ }^{2}$ Chance Developmental Support Center, China \\ Correspondence: Guofu Zhang, Wuxi Mental Health Center, Wuxi Tongren International Rehabilitation Hospital, \\ Nanjing Medical University, Wuxi, China. Tel: 852-9324-4185. E-mail: zhangguofuwx@163.com
}

Received: January 18, 2016

Accepted: January 21, 2016

Online Published: February 29, 2016

doi:10.20849/aes.v1i1.34

URL: http://dx.doi.org/10.20849/aes.v1i1.34

\begin{abstract}
Chinese Version Sensory Profile (Cheung \& Siu, 2009) is a validated tool for healthcare professionals to analyse the impact on how sensory impairment may affect children in carrying out daily activities.

In this study, a total of 15 caregivers were recruited from a special school for mental handicap in Hong Kong to complete a survey in respect of the children's condition (11 males and 4 females aged 6 to 15 years old).

Results showed that the proportion falling on imbalance and dysfunction category was highest in visual processing $(66.6 \%)$ and lowest in emotional/social responses $(26.6 \%)$. In order for children with sensory impairments to engage in daily life and learning through the process, a series of activities are recommended to parents and teachers.
\end{abstract}

Keywords: Sensory profile

\section{Introduction}

The Chinese Version Sensory Profile (Cheung \&Siu, 2009) is a standardized assessment tool (validated in Hong Kong) used by health care professionals to analyse the impact on how sensory impairment can affect children in carrying out daily activities. It provides a more holistic view when used alongside other assessment tools and clinical observations for each participant's sensory issues thus incorporates better strategies for follow up interventions.

The Profile looks into 100 components and needs to be completed by the main caregiver of the child who should be familiar with the child's daily routines and how he performs in his daily activities. Each of the sections reflects on the child's reaction to various stimuli. The eight sections are as follows:

1. Auditory processing

2. Visual processing

3. Activity level

4. Taste / smell processing

5. Body position

6. Movement

7. Tactile processing

8. Emotional / social processing

As compared with peer of the same age, the caregiver completes the questions based on how frequently (in a five-point Likert scale from "always", "usually", "occasionally", "seldom" to "never") these described behaviours are observed in the child currently (or in the recent past half year). Occupational therapist can therefore analyse and evaluate on the child's difficulties in processing sensory information. 


\section{Method}

A total of 15 caregivers were selected from a special school for mental handicap in Hong Kong to complete a survey in respect of the children's condition.

\section{Results}

The subjects were 15 children (11males and 4 females, aged 6 to 15). Table 1 shows the proportions of imbalance and dysfunction categories respectively and their sum in each domain of the Profile in the subjects.

The results indicated that the sum of proportion of imbalance/dysfunction was highest in visual processing $(66.6 \%)$ and lowest in emotional/social responses (26.6\%).

The proportion of imbalance was higher than that of dysfunction in five domains ("visual processing", "auditory processing", "tactile processing", "body position" and "movement"). The difference was more significant in three of them which were "visual processing", "tactile processing" and "movement". Another two domains ("taste/smell processing", "activity level") showed the opposite and a more significant difference was found in the latter. The same ratio in imbalance and dysfunction was noted in "Emotional/social processing".

One of the children was indicated dysfunction in all domains and four of them did not appear to have any imbalance/dysfunction. Another two children had problems in only one domain (one with imbalance in auditory processing and the other with dysfunction in activity level). The other eight children were spread into all domains with normal, imbalance or dysfunction.

From the above information, visual processing difficulties were the most common among these children. Further investigation is required to discover the reasons and how this may be affecting them in participating in everyday tasks so as to tailor make interventions.

\section{Recommendation of Activities}

Visual, tactile and proprioceptive processing difficulties were revealed in the participants. Recommendations of exercise/activities should be provided to parents/teachers in order to help regulate sensory information for their children in participating in daily activities.

Visual stimuli (if children indulge in strong visual input, e.g. moving finger or object closely in front of their eyes)

- Use visual contrast and pictures to guide them while learning and at work.

- Avoid them from sitting facing the wall or alone in a corner.

- Invent interesting and fascinating visual sensory integration games/activities (e.g. computer games, visual tracking games) to satisfy their desires in visual input thus replace those non-productive behaviours

Table 1. Proportions of imbalance category and dysfunction category and their sum in each domain

\begin{tabular}{lccc}
\hline Domains & Imbalance (\%) & Dysfunction (\%) & Sum (\%) \\
\hline Visual processing & 53.3 & 13.3 & 66.6 \\
Auditory processing & 26.7 & 20.0 & 46.7 \\
Tactile processing & 40.0 & 6.7 & 46.7 \\
Taste/smell processing & 20.0 & 26.7 & 46.7 \\
Body position & 33.4 & 20.0 & 53.4 \\
Movement & 26.7 & 6.7 & 33.4 \\
Activity level & 6.7 & 40.0 & 46.7 \\
Emotional/social processing & 13.3 & 13.3 & 26.6 \\
\hline
\end{tabular}

Tactile stimuli (if children indulge in strong tactile input, e.g. ripping finger tips, playing with fingers or saliva)

- Invent interesting and fascinating tactile sensory integration games/activities (e.g. playing guitar, doing domestic tasks with rough towel) to satisfy their desires in visual input thus replace those non-productive behaviours

- Perform body brushing and towel exercise 
- Use gloves or spray tape on children to minimise self-stimulatory behaviour

Proprioceptive stimuli (if children keep seeking behaviours in proprioception, e.g. self-destructive behaviour, throwing objects, jumping, pushing people, running or heading the wall)

- Participate in proprioceptive sensory integration activities regularly (e.g. running, climbing, trampolining, loading heavy objects, swimming and pressure massage)

- Design a chart to record their behaviour and give them the right awards they deserve

For those rated as "dysfunction", they are advised to take part in further sensory assessments and treatments with occupational therapists.

\section{Reference}

Cheung, P. P. P., \& Siu, A. M. H. (2009). Chinese Sensory Profile: User's Manual. Hong Kong: Hong Kong Occupational Therapy Association.

\section{Copyrights}

Copyright for this article is retained by the author(s), with first publication rights granted to the journal.

This is an open-access article distributed under the terms and conditions of the Creative Commons Attribution license (http://creativecommons.org/licenses/by/3.0/). 